\title{
Liquid-phase and multiphase hydrodehalogenation of halobenzenes over Pd/C: Reaction selectivity and inhibition/promotion effects by the quaternary salt
}

\author{
Sergei S. Zinovyev ${ }^{\mathrm{a}}$, Alvise Perosa ${ }^{\mathrm{a}, \mathrm{b}}$, Pietro Tundo ${ }^{\mathrm{b}, *}$ \\ a Interuniversity Consortium "Chemistry for the Environment" (INCA), Dorsoduro 2137, 30123 Venezia, Italy \\ ${ }^{\mathrm{b}}$ Ca' Foscari University of Venice, Dorsoduro 2137, 30123 Venezia, Italy \\ Received 22 December 2003; revised 28 April 2004; accepted 29 April 2004 \\ Available online 5 June 2004
}

\begin{abstract}
Hydrodehalogenation of iodo-, bromo-, and chlorobenzene on 5\% Pd/C is studied in an ethanolic base solution and in the aqueous baseisooctane system at $50{ }^{\circ} \mathrm{C}$ and atmospheric pressure of hydrogen. Rates and selectivity of competitive and separate reactions of different halides and the effects due to the quaternary ammonium salt, Aliquat 336, are discussed. The reactivity of halides follows the order PhCl $\approx$ $\mathrm{PhBr}>\mathrm{PhI}$ in the biphasic system. Aliquat 336 promotes hydrodechlorination, but inhibits hydrodebromination and hydrodeiodination. The rate inhibition in the reaction of $\mathrm{PhI}$ is connected to formation of the iodide-substituted ammonium salt, which holds inside $\mathrm{Pd} / \mathrm{C}$ and reinforces poisoning by $\mathrm{I}^{-}$, while the presence of the excess of Aliquat 336 prevents inhibition. In competitive experiments, the phenyl halides react selectively one after another according to the order of their adsorption on $\mathrm{Pd}$ : $\mathrm{PhI}, \mathrm{PhBr}, \mathrm{PhCl}$, where hydrodechlorination and hydrodebromination are slowed down by the iodide anion formed in hydrodeiodination.
\end{abstract}

(c) 2004 Elsevier Inc. All rights reserved.

Keywords: Palladium on charcoal; Hydrodehalogenation; Halobenzenes; Aliquat 336; Inhibition by iodide

\section{Introduction}

Catalytic hydrodehalogenation of halogenated aromatic compounds is a reaction of both practical and theoretical importance. It has a wide use in organic synthesis, and research on the transformation of halogenated aromatics into the corresponding hydrocarbons is also pursuing environmental goals, namely the conversion of toxic polychlorinated and brominated substances into nontoxic products. The basic issues, in this connection, refer to the development of new systems, catalysts, promoters, and hydrogen donors, as well as to the investigation of the poisoning/deactivation of the catalyst.

The use of the liquid-phase systems for hydrodehalogenation, normally in ethanol, has recently received wide popu-

\footnotetext{
* Corresponding author. Dipartimento di Scienze Ambientali, Università Ca' Foscari, Dorsoduro 2137, 30123 Venezia, Italy. Fax: +39 (041) 234 8620.

E-mail address: tundop@unive.it (P. Tundo).
}

larity, because of the mild conditions involved and the high selectivity achievable using hydrogen gas [1-5] or other hydrogen donors [2,6-8]. Typically, various $\mathrm{Pd}$ and $\mathrm{Ni}$ catalysts are considered the most active in this reaction.

We have previously reported on a number of applications of multiphase catalytic systems in the hydrodehalogenation with hydrogen on $\mathrm{Pd} / \mathrm{C}$ [9-11] and Raney-Ni [11,12] of various halobenzenes, including polyhalogenated and other substituted ones [13-15], as well as toxic chlorinated substances, such as polychlorinated biphenyls [10] and dioxins [16]. The multiphase system operates at $50^{\circ} \mathrm{C}$ and atmospheric pressure of hydrogen and consists of a biphasic hydrocarbon/aqueous base medium, containing also a quaternary ammonium salt, e.g., Aliquat 336 (tricaprylmethylammonium chloride). The latter forms a separate liquid layer, coating the heterogeneous catalyst, and acts as promoter (in the hydrodechlorination reaction) or as a catalyst modifier which often allows changing the reaction chemo-, regio-, or enantioselectivity (in the reduction of other functional groups and polyfunctional substrates) [17]. 
In previous studies we have pointed out that Aliquat 336 (tricaprylmethylammonium chloride), as well as other quaternary ammonium salts, significantly increase the hydrodechlorination rate and selectivity under multiphase conditions. However, the reduction of aromatic iodine is, instead, slowed down in the presence of Aliquat 336 [10]. This phenomenon is ascribed to the substitution of iodide for chloride anion in the onium salt QCl. The resulting QI is assumed to be adsorbed on the catalyst surface that reinforces the poisoning effect of $\mathrm{I}^{-}$on Pd [10].

The reactivity order of phenyl halides under the multiphase conditions, $\mathrm{PhBr}>\mathrm{PhCl}>\mathrm{PhI}$, is also in agreement with the more recent results reported by Aramendía et al. for the hydrodehalogenation of halobenzenes with hydrogen on Pd in ethanol [2]. It has been suggested in [2] that the reactivity of halobenzenes stems from the conjunction of both the dissociation energy of the $\mathrm{C}-\mathrm{X}$ bond (the weaker bond must induce faster reaction) and the adsorption strength of the reagent: the adsorption of $\mathrm{PhI}$ on $\mathrm{Pd}$ is considered as excessive, which results in low activity. Wiener et al. have also reported [7] that the adsorption of iodobenzene is so strong that it may render the catalyst surface inaccessible to the hydrogen donor.

Interestingly, in the above-noted work of Aramendía et al. the reactivity of halides decreases in the order $\mathrm{PhI}>\mathrm{PhBr}>$ $\mathrm{PhCl}$ if using 2-propanol as the hydrogen donor, whereas in the hydrodehalogenation with potassium formate, reported by Wiener et al., chlorobenzene is more reactive than bromobenzene. According to the results of [18] the reactivity of halides decreases in the order $\mathrm{PhCl}>\mathrm{PhBr}>\mathrm{PhI}$, i.e., exactly opposite to that corresponding to the true reactivity order of $\mathrm{C}-\mathrm{X}$ bonds. From the evidence, various factors may affect the reactivity of halides, among which could be solvent effects, adsorption strength, inhibition phenomena, etc.

It has been pointed out in several studies $[2,7,18,19]$ that the adsorption strength of phenyl halides in the hydrodehalogenation reaction plays an important role and can strongly affect the order of competitive reactions of different halides or inhibit the reduction of other groups, either present in different substrates or in the same molecule. The competitive reactions of halobenzene mixtures are consecutive, and usually follow the order $\mathrm{PhI}, \mathrm{PhBr}, \mathrm{PhCl}$, as was observed under different reaction conditions and with different hydrogen sources (e.g., with hydrogen and 2-propanol) [2,9]. Particular attention is paid to the high "consecutive selectivity" of such reactions. For example, the reduction of aromatic iodine can be carried out very selectively with respect to the reduction of other groups, sensible to reduction. Faucher et al. have recently demonstrated a practical advantage of this selectivity for the synthesis of tritium or deuterium-substituted organic compounds [18]. Aromatic iodine is substituted with $\mathrm{D}_{2}$ or $\mathrm{T}_{2}$ over $\mathrm{Pd} / \mathrm{C}$ with up to $98 \%$ selectivity, leaving other functionalities, such as double bonds, carbonyl, benzyloxy-, nitro-, and some other groups unreduced.

The present study is focused on the kinetics of hydrodehalogenation of different halobenzenes with hydrogen gas over $\mathrm{Pd} / \mathrm{C}$ and is aimed at understanding the effect produced by the nature of the halide anion on the reaction. The effect of halide species appears to be vital in determining the catalytic activity and selectivity of hydrodehalogenation in the multiphase system, particularly because of the presence of the quaternary salt $\mathrm{QX}$, which can participate in the anion exchange. In fact, the presence of the QX in the immediate vicinity to the catalyst surface makes the anion influence more pronounced. The multiphase system by its nature and mechanism of action is different from the classical hydrodehalogenation systems (where alcohols are usually employed as solvents). In this connection, this study draws a comparison between ethanolic and multiphase systems. Both competitive and separate reactions of halobenzenes are considered.

\section{Experimental}

All reagents and solvents were used as purchased and no further purification was done. A $5 \% \mathrm{Pd} / \mathrm{C}$ was from Aldrich, Art. No. 20,568-0. Aliquat 336 (tricaprylmethylammonium chloride) was from Aldrich, Art. No. 20,561-3. GC analyses were performed on a Varian 3400 using a fused-silica capillary column Chrompack CP-Sil 24 CB lowbleed/MS $(30 \mathrm{~m} \times 0.25 \mathrm{~mm}$, film thickness $0.25 \mu \mathrm{m})$.

The reactions were run in a $25-\mathrm{mL}$ three-necked roundbottomed reactor with a water jacket, supplied with a tube for hydrogen supply, and a condenser, stirred magnetically at $1000 \mathrm{rpm}$, and thermostated at $50 \pm 1^{\circ} \mathrm{C}$. The reaction was loaded with a $10-\mathrm{mL}$ solution in isooctane $(1: 1 \mathrm{vol})$, or in absolute ethanol, of $0.7 \mathrm{mmol}$ of a substrate (chloro-, bromo-, or iodobenzene), or of a mixture of $0.7 \mathrm{mmol}$ of each (in competitive experiments), of $0.059 \mathrm{~g}$ of $n$-dodecane $(0.35 \mathrm{mmol})$ as the GC internal standard and, where indicated, of $0.103 \mathrm{~g}(0.26 \mathrm{mmol})$ or more (see legends to figures) of Aliquat 336, and contained also $0.0455 \mathrm{~g}$ of $5 \%$ $\mathrm{Pd} / \mathrm{C}(0.02 \mathrm{mmol} \mathrm{Pd})$. The amount of $5.7 \mathrm{~mL}$ of $10 \%$ aqueous $\mathrm{KOH}$ was added therein if isooctane was the organic phase, or $0.01 \mathrm{~mol}$ of $\mathrm{KOH}$ was dissolved in ethanol, if the latter was the solvent. The reaction was bubbled with $\mathrm{H}_{2}$ at atmospheric pressure at $10 \mathrm{~mL} / \mathrm{min}$.

The samples were collected from the organic phase during the reaction at time intervals. The first one was taken just after the reagents had been loaded and it was considered the zero-time point. About $20 \mu \mathrm{L}$ of the reaction mixture was taken for each sample, which was then diluted up to 1-2 mL with ethyl ether or with ethanol, if the latter was the solvent. To avoid GC column pollution/damage and to enhance the quality of analyses, the samples were shaken with silica to remove Aliquat 336 and/or filtered with glass wool to remove catalyst. The samples in ethanol that contained $\mathrm{KOH}$ were neutralized with aqueous $\mathrm{HCl}$.

The quantitative analyses of the reaction mixture components were performed using the GC internal standard technique, as described elsewhere [12]. Calibration coefficients 
for $\mathrm{PhI}, \mathrm{PhBr}, \mathrm{PhCl}$, and benzene were determined to be $0.072,0.077,0.078$, and 0.076 , respectively.

The fitting of experimental data (concentration vs time) to calculate the rate constants was performed by means of the Microcal Origin v. 6.0 software.

\section{Results and discussion}

\subsection{Competitive reactions of phenyl halides}

Hydrodehalogenation of a mixture of phenyl halides (PhI, $\mathrm{PhBr}, \mathrm{PhCl}$, and $\mathrm{PhF}$ ) with hydrogen over 5\% $\mathrm{Pd} / \mathrm{C}$ has been studied in different solvent systems (ethanol and the biphasic isooctane/aqueous medium) with and without the catalyst modifier, Aliquat 336. We have found that no halide in the mixture reacted in the absence of base, in ethanol, or in isooctane, or in the biphasic isooctane/water system.

Reacting the mixture of halides under the same conditions in the ethanolic $\mathrm{KOH}$ solution or in the isooctane/aqueous $\mathrm{KOH}$ system results in the exhaustive hydrodehalogenation of chloro-, bromo-, and iodobenzenes to benzene. Fluorobenzene does not react under the conditions studied; its disappearance from the mixture is caused by its evaporation, as was proven by setting an independent experiment with $\mathrm{PhF}$ (no benzene is formed). The other halides react with high rates and with a surprising "consecutive" selectivity; i.e., the reaction of iodobenzene selectively precedes that of bromobenzene, and the latter, in turn, is totally converted to benzene before chlorobenzene starts to react (the reaction profiles in isooctane-aqueous $\mathrm{KOH}$ are presented in Fig. 1; similar sequence of profiles was observed in the ethanol$\mathrm{KOH}$ system). This phenomenon, which we have previously described briefly elsewhere [9], is probably connected to a difference in the affinity of these substrates to the Pd catalyst surface [7].

In the system with isooctane and $10 \%$ aqueous $\mathrm{KOH}$, as well as in most other cases discussed below, the reaction profiles for all halobenzenes could be described well by zero-order kinetics (Fig. 1). Even though the adsorption strengths for different halides on the catalyst must be very different, the initial rates appeared quite similar (Fig. 1, the corresponding zero-order rate constants are reported in Table 1). In the case of iodobenzene, however, the rate decreases fourfold after reaching nearly half of conversion. The observed zero-order kinetics and the selective order of

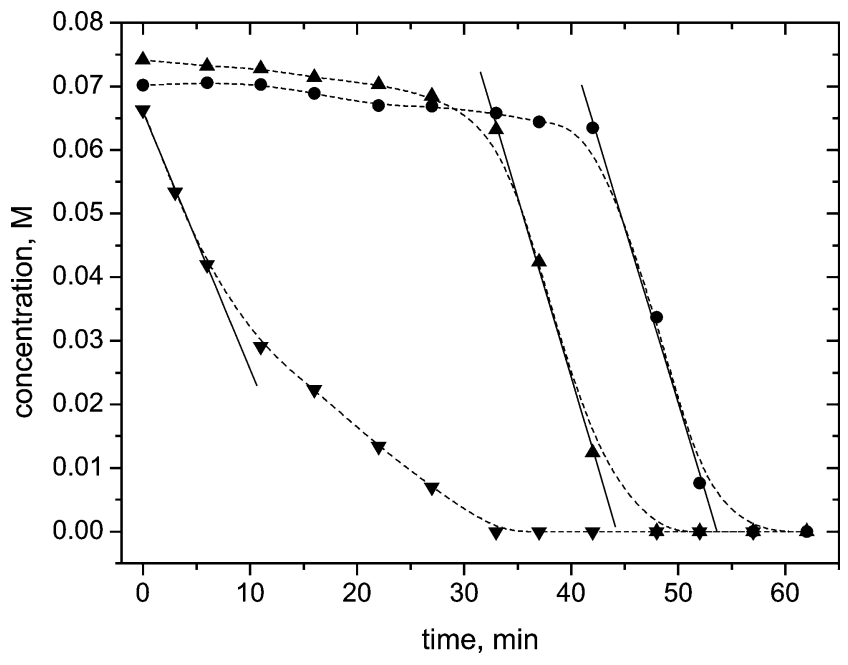

Fig. 1. Hydrodehalogenation of a mixture of $\mathrm{PhI}(\boldsymbol{\nabla}), \mathrm{PhBr}(\boldsymbol{\Delta})$, and $\mathrm{PhCl}$ (O) with $\mathrm{H}_{2}$ over $5 \% \mathrm{Pd} / \mathrm{C}$ in the isooctane/10\% aqueous $\mathrm{KOH}$ system (in the absence of Aliquat 336). Symbols and dashed lines refer to experimental data and smoothing; solid lines, a linear approximation of the experimental data at given intervals of concentration (for rate constants see entry 1 , Table 1).

reactivity of halides suggest that the catalyst surface is saturated by the reacting species within the overall reaction time. Hence, the substrate adsorption must not be rate limiting. The reaction is probably limited by one of the processes on the surface that follow adsorption, e.g., reaction with hydrogen, desorption of products, or neutralization of hydrogen halide formed, which has an inhibition effect on the reaction [20-22]. Some slowing down of the reaction of iodobenzene (Fig. 1) could be due to a poisoning of the catalyst by the released iodide anion [10], which competes with the substrate for the catalyst surface.

The same order of reactions of phenyl halides is observed in the ethanol $\mathrm{KOH}$ solution (rate constants reported in Table 1). Both hydrodeiodination and hydrodebromination reactions exhibit very high and comparable rates, though the reaction of chlorobenzene proceeds with a rate one order of magnitude slower than that of other halides, and also slower than the rate of chlorobenzene reaction in the biphasic system. Nevertheless, the separate reaction of chlorobenzene in ethanol manifests a high rate, similar to those of other halides (entry 4, Table 1), which suggests that the presence of $\mathrm{KI}$ and/or $\mathrm{KBr}$, formed in the reactions of bromo- and iodobenzenes, inhibits the reaction of chlorobenzene [10]. This inhibition seems to be more pronounced in the ethanol

Table 1

Rate constants of the hydrodehalogenation with $\mathrm{H}_{2}$ over $5 \% \mathrm{Pd} / \mathrm{C}$ for competitive and separate reactions of halobenzenes in different systems

\begin{tabular}{|c|c|c|c|c|c|}
\hline \multirow[t]{2}{*}{ No. } & \multirow[t]{2}{*}{ System } & \multirow[t]{2}{*}{ Type } & \multicolumn{3}{|c|}{ Zero-order rate constant $\left(\mathrm{mol} \mathrm{L}^{-1} \mathrm{~min}^{-1}\right) \times 10^{3}$} \\
\hline & & & $\mathrm{PhI}$ & $\mathrm{PhBr}$ & $\mathrm{PhCl}$ \\
\hline 1 & $i$-Octane/10\% aq KOH & Competitive & $4.05 \pm 0.14$ & $5.66 \pm 0.23$ & $5.54 \pm 0.43$ \\
\hline 2 & Ethanol, $1.0 \mathrm{M} \mathrm{KOH}$ & Competitive & $11.33 \pm 0.45$ & $8.82 \pm 1.11$ & $0.93 \pm 0.06$ \\
\hline 3 & $i$-Octane $/ 10 \%$ aq $\mathrm{KOH}$ & Separate & $0.48 \pm 0.02$ & $22.71 \pm 0.1$ & $21.89 \pm 0.38$ \\
\hline 4 & Ethanol, $0.84 \mathrm{M} \mathrm{KOH}$ & Separate & - & - & $7.2 \pm 0.01$ \\
\hline
\end{tabular}


Table 2

Hydrodechlorination and hydrodebromination rate constants of different halogenated substrates in their reactions with $\mathrm{H}_{2}$ over $\mathrm{Pd} / \mathrm{C} 5 \%$ in the ethanolic base $(\mathrm{KOH} 0.84 \mathrm{M})$ solution

\begin{tabular}{|c|c|c|c|}
\hline \multirow[t]{2}{*}{ No. } & \multirow[t]{2}{*}{ Substrate } & \multicolumn{2}{|c|}{$\begin{array}{l}\text { Zero-order rate constant } \\
\left(\mathrm{mol} \mathrm{L}^{-1} \mathrm{~min}^{-1}\right) \times 10^{3}\end{array}$} \\
\hline & & Dechlorination & Debromination \\
\hline 1 & Chlorobenzene $^{\mathrm{a}}$ & $7.2 \pm 0.01$ & \\
\hline 2 & Bromobenzene $^{\mathrm{b}}$ & & $8.8 \pm 1.1$ \\
\hline 3 & $p$-Chlorobromobenzene & $8.0 \pm 1.0^{\mathrm{c}}$ & $\sim 8.0^{\mathrm{c}}$ \\
\hline 4 & $p$-Bromo-tert-butylbenzene & & $\sim 5.0$ \\
\hline
\end{tabular}

solution where iodide and bromide are more concentrated, whereas in the biphasic system the major part of them stays dissolved in the aqueous phase.

The order of reactions of halobenzenes $(\mathrm{PhI}>\mathrm{PhBr}>$ $\mathrm{PhCl}$ ) as noted above seems to be controlled by the adsorption strengths of these substrates on Pd/C. In the reaction of $p$-chlorobromobenzene on $\mathrm{Pd} / \mathrm{C}$ in ethanol, hydrodebromination and hydrodechlorination steps are consecutive, as was also observed in [23]; that is, the removal of bromine precedes that of chlorine, giving rise to chlorobenzene as the only reaction intermediate. The observed dechlorination and debromination rates are close (Table 2) and correspondent to the values observed in the separate reaction of chlorobenzene and both the competitive and the separate reaction of bromobenzene, respectively (Tables 1 and 2).

These data suggest that the adsorption of halobenzenes involves the coordination of the carbon-halogen bond rather than that of the aromatic ring. This is in contrast to the expectations that could be derived from the HREELS studies on benzene adsorption, which suggest a flat configuration of benzene adsorption on Pd $\{111\}$ [24]. However, the recent XPS and NEXAFS data on benzene adsorption on Pd $\{111\}$ [25] suggest the possibility of a tilted geometry of benzene adsorption at higher surface coverage; i.e., the flat coordination of aromatics on Pd surface may be not so critical. The flat adsorption of $p$-chlorobromobenzene via the formation of $\pi$-complex with the aromatic ring or via the coordination of both $\mathrm{C}-\mathrm{Cl}$ and $\mathrm{C}-\mathrm{Br}$ bonds seems to be unlikely, because both these bonds would be exposed to the reaction with hydrogen; and a mixture of chloro- and bromobenzenes would be formed. Our results are more in agreement with the hypothesis of dissociative adsorption of halobenzenes via the $\sigma$-complex of $\mathrm{Ph}-\mathrm{Hal}$ with the catalyst active site, as reported in [26]. If the adsorption of a halobenzene via $\sigma$-complex is indeed dissociative, the geometry of the aromatic ring adsorption might no longer be important, because the dissociation of only one $\mathrm{C}-\mathrm{Hal}$ bond appears possible at a time. However, the electron affinity of $\mathrm{Br}$ is lower than that of $\mathrm{Cl}$, which is thought in [27] to be a reason for lower reactivity of $\mathrm{PhBr}$ relative to $\mathrm{PhCl}$ in the gas-phase system on $\mathrm{Ni} / \mathrm{SiO}_{2}$. In our case, the $\mathrm{C}-\mathrm{Cl}$ bond in $p$-chlorobromobenzene is reduced only after the $\mathrm{C}-\mathrm{Br}$ one, which discards the possibility of dissociative adsorption. Thus, we suppose that the adsorption of $p$-chlorobromobenzene on $\mathrm{Pd}$ in the liquid-phase system takes place exclusively via the $\sigma$-coordination of the $\mathrm{C}-\mathrm{Br}$ bond, where the aromatic ring is possibly in tilted configuration, and that the adsorbed species occupy all the available active sites (the catalyst surface is saturated by such adsorbed species) so that only one reaction is possible at a time, namely hydrodebromination the first and hydrodechlorination the second.

Another fact, suggesting that the adsorption of the aromatic ring via $\pi$-complex is not critical, can be derived from the consideration of $p$-bromo-tert-butylbenzene reaction under the same reaction conditions. The presence of a bulky tert-butyl group on the aromatic ring, which is expected to restrict interaction of the aromatic ring with the catalyst site, does not produce much effect; the hydrodebromination rate (Table 2) observed in this case is only slightly lower than that in the reactions of $p$-chlorobromobenzene or bromobenzene.

\subsection{Competitive hydrodehalogenation in the multiphase system with Aliquat 336}

By analogy, the reaction of a mixture of halides on $\mathrm{Pd} / \mathrm{C}$ has been carried out in the isooctane/aqueous $\mathrm{KOH}$ system in the presence of Aliquat 336 (taken at 37\% amount with respect to each substrate, or $0.026 \mathrm{~mol} / \mathrm{L})$. The sequence of the reactions of different halides remains the same as in the previously discussed case, i.e., from iodide to chloride (Fig. 2). However, the reaction of iodobenzene, which proceeds rapidly in the beginning, is then strongly inhibited after reaching nearly $30 \%$ conversion. A subsequent addition of $\mathrm{KOH}$ does not restore the initial rate. On the other hand, the addition of two more equivalents of Aliquat 336 results in a resumption of the original rate. The subsequent reactions of bromo- and chlorobenzenes are characterized by far

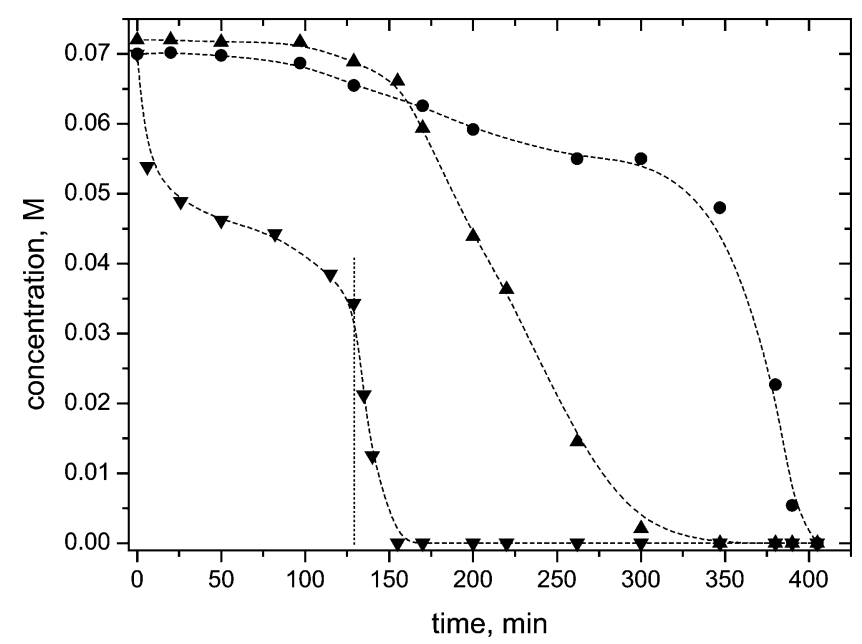

Fig. 2. Hydrodehalogenation of a mixture of $\mathrm{PhI}(\boldsymbol{\nabla}), \operatorname{PhBr}(\boldsymbol{\Delta})$, and $\mathrm{PhCl}(-)$ with $\mathrm{H}_{2}$ over $5 \% \mathrm{Pd} / \mathrm{C}$ in the isooctane/10\% aqueous $\mathrm{KOH}$ system in the presence of Aliquat $336(0.26 \mathrm{mmol}$, and then $0.5 \mathrm{mmol}$ was added at a 130 -min reaction time). 
lower rates than in the system without Aliquat 336 (compare Figs. 1 and 2).

In our system the chloride counterion of Aliquat 336 $(\mathrm{QCl})$ is easily substituted with the iodide anion, released during hydrodeiodination, to give tricaprylmethylammonium iodide (QI) [28]. Independent tests have shown that in the isooctane/aqueous $\mathrm{KOH}$ system with $\mathrm{QCl}$ the formation of a visually identified separate yellow layer between aqueous and organic phases takes place upon addition of KI. This separate layer is mainly composed by QI, which is formed via the anion exchange of $\mathrm{QCl}$ with $\mathrm{KI}$. It is also noticeable that the QI phase, present at the interface in the biphasic system, is poorly dispersed upon stirring and tends to coat the particles of supported catalyst, thereby causing their aggregation within its layer. Contrarily, $\mathrm{QCl}$ improves dispersion of the catalyst particles. In the experiment reported in Fig. 2, the dispersion of catalyst was visually deteriorating after the time point corresponding to the beginning of rate inhibition, and right after a new portion of $\mathrm{QCl}$ had been added (130 min in Fig. 2) and the rate had been restored, the good catalyst dispersion was attained again.

In this sense, the rate inhibition of iodobenzene reduction in the presence of Aliquat 336 can be ascribed to the poor catalyst dispersion, though this does not exclude a possible poisoning of the catalyst surface by the iodide anion, which coats the catalyst, once coupled with the quaternary cation. In order to understand in more detail the nature of rate inhibition and the role of Aliquat 336 in this connection, a series of experiments has been conducted with iodobenzene as the only substrate.

\subsection{Hydrodeiodination in the multiphase system-Effect of Aliquat 336}

Hydrodeiodination of iodobenzene in the isooctane/aqueous $\mathrm{KOH}$ system on $\mathrm{Pd} / \mathrm{C}$ in the presence of Aliquat 336 proceeds with a high rate up to a conversion which roughly corresponds to the concentration of Aliquat 336 present $(0.26 \mathrm{~mol} / \mathrm{L})$ (Fig. 3). After that, as well as in the competitive experiment, the rate falls noticeably. We have found that in the "slow period" the reaction proceeds with a rate equal to that of the reaction, which is started with tricaprylmethylammonium iodide instead of Aliquat 336 (the reaction is loaded with 1 eq of Aliquat 336 and a slight excess of KI, see Fig. 3). One can see from Fig. 3 that upon a threefold increase of Aliquat 336 concentration in the system, a corresponding increase of conversion is observed in the initial fast period, and in the presence of 5 eq of Aliquat 336 (exceeds the substrate amount) the reaction proceeds with no sign of inhibition and is considerably faster than in the absence of Aliquat 336 (Fig. 4).

It must be noted that in the system without Aliquat 336 an addition of KI to the reaction of iodobenzene (Fig. 4) does not affect the rate, and that, in this case, the rate is higher than in the experiment where Aliquat 336 and $\mathrm{KI}$ are present together, but lower than that of the initial period of reactions

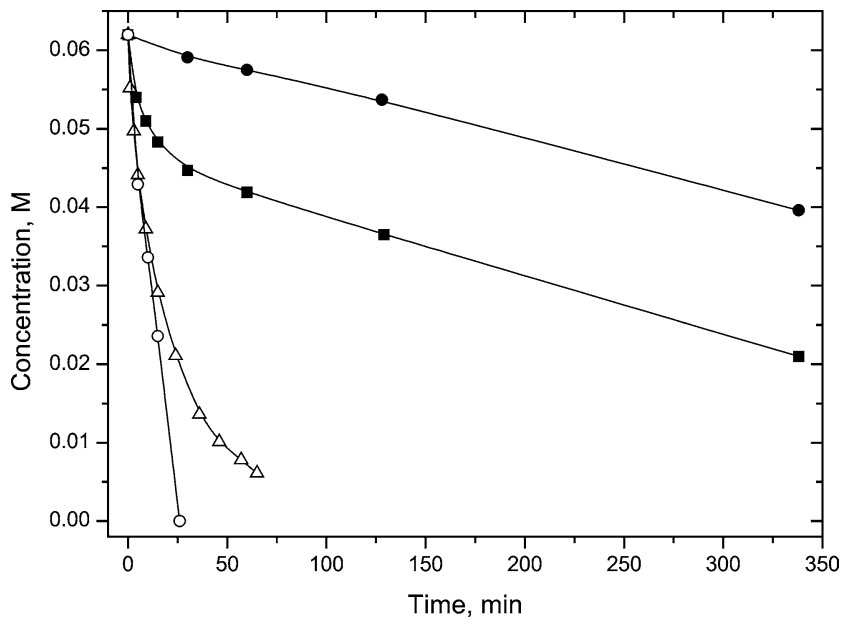

Fig. 3. Reaction of iodobenzene with $\mathrm{H}_{2}$ over $5 \% \mathrm{Pd} / \mathrm{C}$ in the isooctane/10\% aqueous $\mathrm{KOH}$ system in the presence of (-) 1 eq of Aliquat 336 $(0.26 \mathrm{mmol})$ and KI $(0.35 \mathrm{mmol})$; (口) 1 eq of Aliquat $336 ;(\triangle) 3$ eq of Aliquat 366; (○) 5 eq of Aliquat 336.

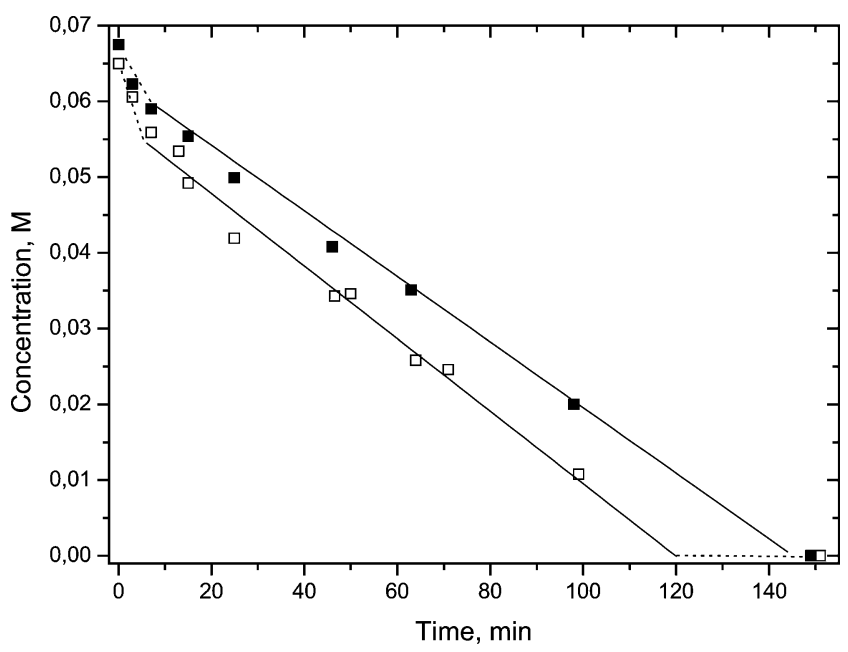

Fig. 4. Reaction of $\mathrm{PhI}$ with $\mathrm{H}_{2}$ over $5 \% \mathrm{Pd} / \mathrm{C}$ in the isooctane/10\% aqueous $\mathrm{KOH}$ system in the absence of A336, with ( $\square$ ) and without ( $\square$ ) KI (0.35 mmol).

in the presence of Aliquat 336 (Fig. 3). Therefore, we can conclude that Aliquat 336 per se promotes the reaction of iodobenzene.

We have noted also that Aliquat $336(\mathrm{QCl})$ is extracted from its solution in isooctane by the QI layer. In this sense, the presence of QCl in the bulk of QI could eventually result in one or more of the following: (1) competitive adsorption on the catalyst surface and, thereby, liberation of a portion of surface, poisoned by $\mathrm{I}^{-}$; (2) anticoagulation effect, and following from this, enhancement of mass transfer (in the presence of the QI-QCl phase a better catalyst dispersion was observed).

One can note that the rate of iodobenzene reaction (alone) in isooctane/aqueous $\mathrm{KOH}$ without Aliquat 366 (Fig. 4) is considerably lower than that in the competitive experiment with the other halides under the same conditions (Fig. 1). The higher rate of iodobenzene reaction in the mixture seems 
to be due to the presence of bromobenzene, as has been evidenced by comparing the kinetic profiles of iodobenzene reaction in mixtures with chloro- and bromobenzene. The reaction rate of iodobenzene in the mixture with chlorobenzene is slow and not different from that of the reaction of iodobenzene alone, while the reaction of iodobenzene in the mixture with bromobenzene proceeds rapidly and has the same rate as in the competitive experiment (Fig. 1), even though the content of $\mathrm{PhBr}$ is nearly 10-fold lower than that of PhI (see Fig. 5).

\subsection{Hydrodechlorination and hydrodebromination reactions}

Contrary to iodobenzene, the separate reactions of chloroand bromobenzene in isooctane/aqueous $\mathrm{KOH}$ without Aliquat 336 are very rapid (see entry 3 of Table 1), and much faster than in the analogous competitive experiment (Fig. 1 and entry 1 of Table 1). This again stresses the idea that the iodide anionic species represent a poison not only for the hydrodeiodination reaction but also for dechlorination and debromination. The presence of Aliquat 336 along with the iodide anion, QI, seems to reinforce the poisoning by the latter also for the reactions chloro- and bromobenzene (Fig. 2).

An inhibition effect similar to that of iodide, but less pronounced, can also be observed due to the release of bromide anion in the reaction of bromobenzene in isooctane/10\% aqueous $\mathrm{KOH}$ (Fig. 6). In the presence of 1 eq of Aliquat 336 there is a rate decrease after a certain conversion (in the initial period the rate is the same as in the experiment without Aliquat 336). In the presence of 3 eq of Aliquat 336, instead, the reaction speeds up and is almost complete in just $1 \mathrm{~min}$ (Fig. 6).

In the separate reaction of chlorobenzene, there is no inhibition at any concentration of Aliquat 336, but only acceleration of the reaction. In fact, the cocatalytic effect by quaternary salts is more pronounced in the hydrodechlorination reaction, as our previous results have shown, particularly for the polychlorinated substrates, which are sometimes impossible to dechlorinate without Aliquat 336, e.g., in the reaction of di- and trichlorobenzenes over Raney-Ni in the isooctane/aqueous $\mathrm{KOH}$ system $[11,12]$.

\section{Conclusions}

(1) The reactions of a mixture of phenyl chloride, bromide, and iodide over $\mathrm{Pd} / \mathrm{C}$ proceed consecutively in the predefined order $\mathrm{PhI}, \mathrm{PhBr}, \mathrm{PhCl}$, which is induced by the order of the adsorption strength of these species on Pd. Seemingly, the adsorption of a halobenzene on $\mathrm{Pd} / \mathrm{C}$ takes place via the $\sigma$-coordination of the carbon-halogen bond and the catalyst is saturated by the adsorbed halobenzene species during the reaction.

(2) The quaternary ammonium salt, Aliquat 336, promotes hydrodehalogenation in the isooctane/aqueous $\mathrm{KOH}$

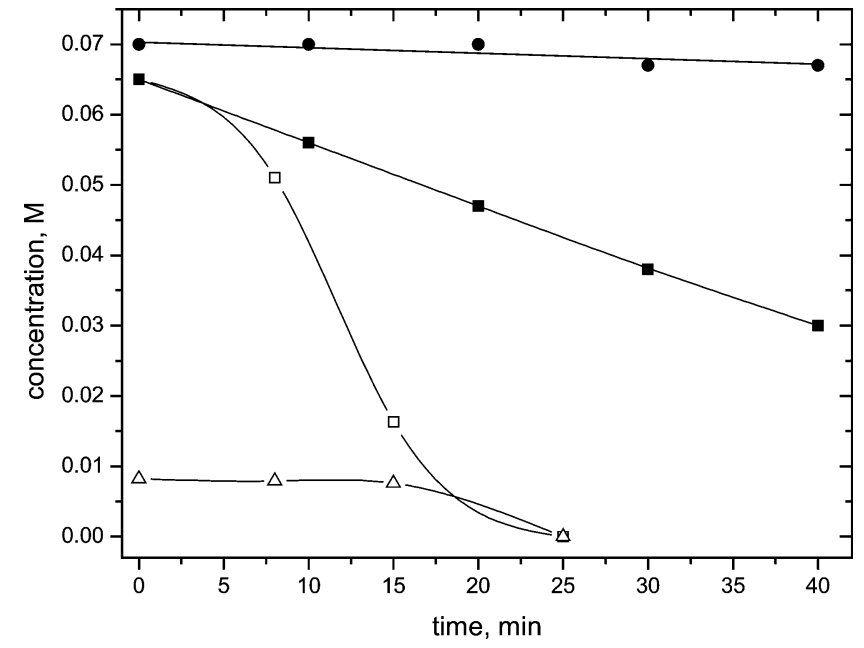

Fig. 5. Reactions of iodobenzene in the presence of chlorobenzene ( iodobenzene; $\bullet$, chlorobenzene) and of bromobenzene ( $\square$, iodobenzene; $\triangle$, bromobenzene) over $5 \% \mathrm{Pd} / \mathrm{C}$ in isooctane/10\% aqueous $\mathrm{KOH}$.

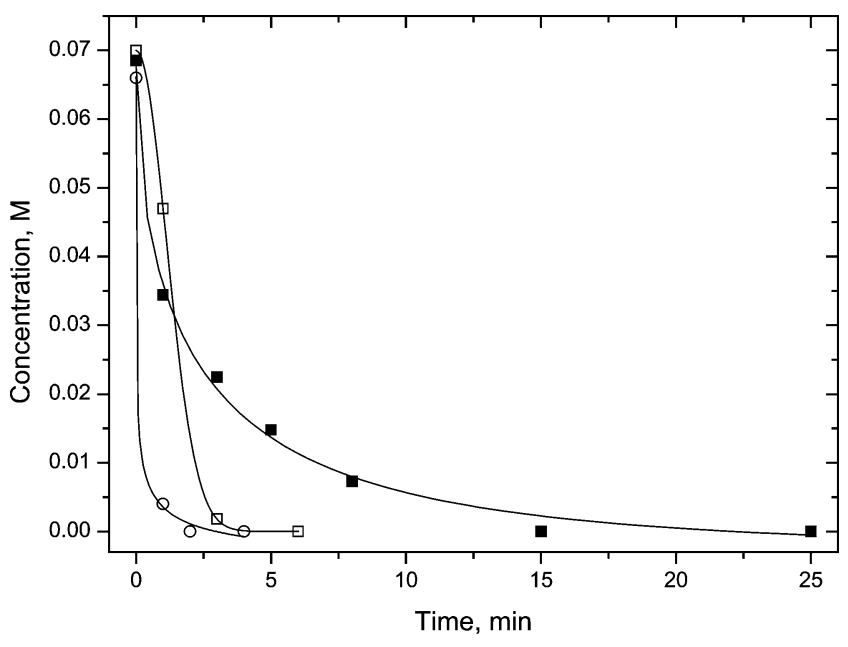

Fig. 6. Reaction of bromobenzene with $\mathrm{H}_{2}$ over $5 \% \mathrm{Pd} / \mathrm{C}$ in the isooctane/10\% aqueous $\mathrm{KOH}$ system vs the amount of Aliquat 336: ( $\square$ ) without Aliquat 336 (see also entry 3, Table 1), (ם) 1 eq of Aliquat $336(0.26 \mathrm{mmol})$, (○) 3 eq of Aliquat 336.

system, where its maximum effect is felt on the hydrodechlorination reaction. However, for the hydrodebromination or hydrodeiodination reactions, the stoichiometric amount or excess of Aliquat 336 is required, since the latter is required to overrun the rate inhibition, caused by the presence of iodide or bromide anions on the catalyst surface.

(3) In the hydrodehalogenation of bromobenzene and iodobenzene, especially in the case of the latter, the inhibition effect on the reaction is produced by the released halide anion. In the reaction of iodobenzene such inhibition can be reinforced due to the presence of the iodide-substituted Aliquat 336. The latter, formed as a result of anion exchange of Aliquat 336 with KI, forms an immiscible liquid layer between aqueous and organic phases and coats the Pd catalyst, thereby making it more accessible by the iodide anionic species. Therefore, the role of the quaternary ammonium 
salt, if taken in insufficient amount, may be negative, i.e., that of an "inhibition promoter."

(4) The maximum inhibition is produced by the iodide anion, because of its high affinity to $\mathrm{Pd}$. This inhibition affects also the reactions of bromo- and chlorobenzene in the mixture of halides.

The clarification of the above-noted effects sheds light on some peculiarities of the mechanism of hydrodehalogenation in the multiphase systems, particularly on how the nature of the halogen, coupled with the presence of quaternary salt, can affect the reaction rate and behavior in both negative and positive ways.

\section{Acknowledgments}

We acknowledge the support of the Italian Ministry of Foreign Affairs, the Italian Interuniversity Consortium "Chemistry for the Environment" (INCA) (Piano "Ambiente Terrestre: Chimica per l'Ambiente," Legge 488/92, Project No. 6), Ca' Foscari University of Venice, INTAS Grant 2000-00710. S. Zinovyev acknowledges funding from INTAS YSF 00-138.

\section{References}

[1] E.N. Balko, E. Przybylski, F.V. Trentini, Appl. Catal. B 2 (1993) 1-8.

[2] M.A. Aramendía, V. Borau, I.M. García, C. Jiménez, A. Marinas, J.M Marinas, F.J. Urbano, C. R. Acad. Sci. Paris Ser. IIc (2000) 465-470.

[3] M.A. Aramendía, R. Burch, I.M. García, A. Marinas, J.M. Marinas, B.W.L. Southward, F.J. Urbano, Appl. Catal. B 31 (2001) 163-171.

[4] E.-J. Shin, M.A. Keane, React. Kinet. Catal. Lett. 69 (2000) 3-8.
[5] C. Schüth, M. Reinhard, J. Appl. Catal. B 18 (1998) 215.

[6] B.T. Khai, A. Arcelli, J. Org. Chem. 54 (1989) 949.

[7] H. Wiener, J. Blum, Y. Sasson, J. Org. Chem. 56 (1991) 6145-6148.

[8] Y. Ukisu, S. Kameoka, T. Miyadera, Appl. Catal. B 27 (2000) 97.

[9] C.A. Marques, M. Selva, P. Tundo, J. Org. Chem. 58 (1993) 5256.

[10] C.A. Marques, M. Selva, P. Tundo, J. Org. Chem. 59 (1994) 3830.

[11] C.A. Marques, O. Rogozhnikova, M. Selva, P. Tundo, J. Mol. Catal. A: Chem. 96 (1995) 301.

[12] S. Zinovyev, A. Perosa, S. Yufit, P. Tundo, J. Catal. 211 (2002) 347.

[13] A. Perosa, M. Selva, P. Tundo, J. Org. Chem. 64 (1999) 3934.

[14] P. Tundo, S. Zinovyev, A. Perosa, J. Catal. 196 (2000) 330.

[15] A. Perosa, P. Tundo, S. Zinovyev, Green Chem. 4 (2002) 492.

[16] P. Tundo, A. Perosa, M. Selva, S.S. Zinovyev, Appl. Catal. B 32 (2001) L1-L7.

[17] P. Tundo, A. Perosa, S.S. Zinovyev, J. Mol. Catal. 204-205 (2003) 747-754.

[18] N. Faucher, Y. Ambroise, J.-C. Cintrat, E. Doris, F. Pillon, B. Rousseau, J. Org. Chem. 67 (2002) 932-934.

[19] Y. Ambroise, C. Mioskowski, G. Djéga-Mariadassuo, B. Rousseau, J. Org. Chem. 65 (2000) 7183-7186.

[20] C.D. Thompson, R.M. Rioux, M. Chen, F.H. Ribeiro, J. Phys. Chem. B 104 (2000) 3067-3077.

[21] J.H. Hoke, G.A. Gramiccioni, E.N. Balko, Appl. Catal. B 1 (1992) 285-296.

[22] M.A. Aramendía, V. Borau, I.M. García, C. Jiménez, M. Lafont, A. Marinas, J.M. Marinas, F.J. Urbano, J. Catal. 187 (1999) 392-399.

[23] M.A. Aramendía, V. Borau, I.M. García, C. Jiménez, A. Marinas, J.M. Marinas, F.J. Urbano, Appl. Catal. B 43 (2003) 71-79.

[24] A. Barbieri, M.A. Van Hove, G.A. Somorjai, Surf. Sci. 306 (1994) 261-268.

[25] A.F. Lee, K. Wilson, R.M. Lambert, A. Goldoni, A. Baraldi, G. Paolucci, J. Phys. Chem. B 104 (2000) 11729-11733.

[26] M.A. Keane, D.Yu. Murzin, Chem. Eng. Sci. 56 (2001) 3185-3195.

[27] C. Park, C. Menini, J.L. Valverde, M.A. Keane, J. Catal. 211 (2002) 451-463.

[28] E.W. Dehmlow, S.S. Dehmlow, Phase Transfer Catalysis, second revised ed., Verlag Chemie GmbH, Weinheim, 1983, p. 14. 